\title{
TEACHING AND LEARNING
}

\section{Leveraging Microsoft Tools for Reference Interactions}

\author{
SALLY ARMSTRONG \\ University of New Brunswick \\ sally.armstrong@unb.ca
}

COVID-19 has greatly impacted library budgets and spending. The acquisition of new tools or software to support library services may now be a luxury that can no longer be afforded. Therefore, librarians need to consider the tools already provided by their library or institution and identify creative ways to leverage them. An example from the University of New Brunswick is the use of the Microsoft 365 suite of services and tools, and more specifically, Microsoft Bookings and Microsoft Teams, for reference interactions.

Microsoft Bookings is a tool that students can easily use to schedule a virtual reference interaction with their librarian. Bookings connects to Microsoft Outlook, which prevents students from doublebooking in your calendar and provides them with a calendar reminder for the meeting. It also connects to Microsoft Teams, which allows you to activate a setting in Bookings that will create a Teams meeting link for every booking created. You can include custom questions for students to answer when they create a booking, such as:

- Please provide a brief description of your project, business idea, or reason for scheduling this meeting.

- What questions can I help you answer?

- Have you done any preliminary research to answer your questions above? Which databases or other resources did you use?

I have also added a question asking whether the meeting is being scheduled for a team, and if the answer is yes, I ask that they provide me with the names and e-mail addresses of their team members. I do this because Bookings does not allow an individual to create a booking for a group. With the contact information, I can simply forward the invitation to the other team members.

Similar to other videoconferencing tools, Teams allows you to share and record your screen, which is very helpful for reference interactions. The difference with Teams, however, is that the recording is uploaded to a shared chat for the meeting, which makes it easy for students to refer back to or share with team members that were unable to attend. The shared chat is also a convenient way to provide links, send files, or ask follow-up questions after the meeting.

There is a small amount of work required to setup a Bookings page. Some basic instructions that take you through the steps of customizing a page can be found in a document created by the author and available at https://unbcloud-my.sharepoint.com/:b:/g/personal/sarmstr4_unb_ca/ Ef5lxoXLbWIKh5bxnRkqp7IBN6F2VQwJw5qD9Ttd_DM9Yw?e=Ba6dgF. 